\title{
INVESTIGAÇÃO CRÍTICA DO DESEMPENHO DO GMRES PRÉ-CONDICIONADO VIA FATORAÇÃO INCOMPLETA LU EM ESTUDOS DE FLUXO DE CARGA
}

\author{
José E. O. Pessanha* \\ pessanha@dee.ufma.br
}

\author{
Carlos Portugal ${ }^{\dagger}$ \\ portugal@ele.puc-rio.br
}

\author{
Osvaldo Saavedra* \\ osvaldo@dee.ufma.br
}

*IEE-UFMA, Avenida dos Portugueses s/n, Campus do Bacanga, São Luís, Ma, - Brasil - 65080-040

${ }^{\dagger}$ PUC/RJ-DEE, Rua Marquês de São Vicente, 225, Gávea, Rio de Janeiro, RJ - Brasil - 22453-900

\begin{abstract}
RESUMO
Este artigo investiga causas associadas ao baixo desempenho de um pré-condicionador ILU e propõe uma metodologia para melhorar a sua qualidade e a taxa de convergência do método iterativo GMRES. O objetivo é solucionar o subproblema linear do fluxo de carga, com destaque para condições de carga pesada e de difícil convergência. A metodologia está baseada em duas etapas. Na primeira, um pré-condicionador é construído a partir da matriz Jacobiana da primeira iteração Newton-Raphson reordenada. A segunda etapa só é considerada se o GMRES falhar no início do processo de solução devido à baixa qualidade do pré-condicionamento. Nela, o processo é reiniciado e um pré-condicionador de melhor qualidade é construído a partir da matriz Jacobiana pré-processada via escalonamento e permutações não-simétricas e simétricas (reordenamento). A fim de amenizar o custo computacional associado a sua construção, uma estratégia para manter o pré-condicionador fixo sempre que possível é incluída. Experimentos numéricos corroboram a robustez numérica e a eficiência computacional da metodologia proposta.
\end{abstract}

PALAVRAS-CHAVE: Métodos Iterativos do Subespaço Krylov, Pré-condicionadores, Equações Algébricas Lineares, Fluxo de Carga.

\footnotetext{
Artigo submetido em 08/04/2009 (ld.: 00991)

Revisado em 02/06/2009, 02/08/2009

Aceito sob recomendação do Editor Associado Prof. Antonio Carlos Zambroni de Souza
}

\begin{abstract}
Critical Investigation of Preconditioned GMRES Via Incomplete LU Factorization Applied to Power Flow Simulation

This paper investigates the causes associated to the low performance of an ILU preconditioner and proposes a methodology to improve its quality and the GMRES iterative method convergence rate as well. The goal is solve the load flow sublinear problem, emphasizing heavy load scenarios of difficult convergence. The methodology is based on two steps. On the first one, a preconditioner is constructed based on the Jacobian matrix calculated and reordered at the first NewtonRaphson iteration. The second step is taking into account only in case of GMRES failure due to low quality preconditioning. The process is restarted an a better quality preconditioner is constructed based on the preprocessed Jacobian matrix through scaling and nonsymmetric and symmetric (reordering) permutations. In order to reduce the computational cost associated to the construction of the preconditioner, it is proposed a strategy for keeping it fixed as long as possible. Numerical experiments corroborate the numerical robustness and the computational efficiency of the proposed methodology.
\end{abstract}

KEYWORDS: Krylov subspace iterative methods, Preconditioners, Algebraic Linear Equations, Load-Flow. 


\section{INTRODUÇÃO}

Como resultado das necessidades inerentes ao crescimento e as exigências atuais dos mercados elétricos, simulações computacionais passaram a envolver sistemas de energia elétrica de grande porte e extremamente complexos em termos de configuração, dispositivos de controle, características e níveis de cargas. Condições de operações muito carregadas e compensadas dificultam a solução das equações matemáticas que representam os modelos, devido, p.ex., ao mau condicionamento da matriz Jacobiana, instabilidade numérica e singularidade. Uma dessas dificuldades pode surgir durante a solução de um fluxo de carga através do método NewtonRaphson, especificamente na solução do subproblema linear do tipo (1).

$$
\text { A. } x=b
$$

Embora métodos diretos, como o baseado em fatoração triangular completa LU (Tinney, 1967) foram, e continuam sendo aplicados para solucionar (1), dependendo do cenário sob investigação estes podem demandar por um esforço computacional excessivo, ou até mesmo fracassar e não solucionar o problema. Para estes casos, métodos iterativos pré-condicionados baseados no subespaço Krylov surgiram como boas alternativas e passaram a ser explorados em diversas áreas da engenharia (Dong \& Li, 2007; Lee et al, 2004; Polydorides et al, 2002; Saitoh \& Kamitani, 2004). Uma das vantagens desses métodos é o alto desempenho e relativa simplicidade de implementação em ambientes computacionais paralelos, fato não observado para os métodos diretos (Alvarado, 1979; La Scala et al, 1989; Chai \& Bose 1993).

Uma proposta inicial em usar métodos iterativos précondicionados em problemas envolvendo sistemas de energia elétrica foi apresentada por (Decker et al, 1992). Os autores combinaram o método LU com o Gradiente Conjugado em análises de estabilidade transitória usando computação paralela. Já a aplicação desta classe de métodos em problemas de fluxo de carga foi apresentada inicialmente por (Galiana et al, 1993), onde os autores fizeram uso do Gradiente Conjugado Pré-condicionado (GCP) no modelo desacoplado rápido e no fluxo cc. Em (Borges et al, 1996) é aplicado o método Gradiente Bi-Conjugado Estabilizado pré-condicionado à solução do problema de fluxo de carga usando Newton-Raphson, e em (Borges et al, 1997) são apresentadas e destacadas as vantagens do uso dos métodos iterativos em ambientes computacionais paralelos. À medida que os métodos iterativos e as técnicas de pré-condicionamento evoluem, novas contribuições surgem, destacando àquelas que utilizam o método Resíduo Mínimo Generalizado Pré-condicionado (GMRES) e suas variantes (ex. GMRES(m), GMRES-E). O desempenho destes métodos é investigado em aplicações envolvendo fluxo de carga (Semlyen, A., 1996; Flueck \& Chiang, 1998), estimação de estado, segurança e estabilidade transitória (Pai \& Dag, 1997).

O presente trabalho propõe uma metodologia para ser empregada na matriz usada na construção de um pré-condicionador baseado em fatoração incompleta (ILU), visando atenuar os problemas de precisão e de estabilidade numérica de seus fatores incompletos e aproveitar as qualidades deste tipo de pré-condicionador. Caso haja solução, a meta é resolver iterativamente através do método GMRES (Saad \& Schultz, 1986) pré-condicionado o subproblema linear do fluxo de carga independentemente das condições de operação. A metodologia consiste em reordenar a matriz Jacobiana da primeira iteração Newton-Raphson e usá-la na construção do pré-condicionador ILU. Se o GMRES falhar já no início do processo de solução devido ao mau-condicionamento dos fatores incompletos, o processo é reiniciado sem a intervenção do usuário e um novo e de melhor qualidade précondicionador é construído a partir da matriz Jacobiana préprocessada via escalonamento e permutações simétricas (reordenamento) e não-simétricas. Estas estratégias já estão bem consolidadas entre os métodos diretos e estão sendo consideradas na construção de pré-condicionadores para métodos iterativos do subespaço Krylov. É incluída na metodologia uma estratégia para reduzir o esforço computacional associado a construção do pré-condicionador a cada nova iteração Newton, mantendo-o fixo sempre que possível durante o processo de solução. Esta estratégia é identificada neste trabalho como pré-condicionamento "Quase-Fixo", ou simplesmente "QF".

Experimentos numéricos com duas configurações do sistema elétrico Brasileiro (2256/3515 barras) operando sob condições de carga pesada e usando-se para fins comparativos um solucionador direto e um pré-condicionador de fatores completos (LU) corroboram a robustez e a eficiência da proposta.

\section{PRÉ-CONDICIONAMENTO}

O desenvolvimento ou adaptação de pré-condicionadores têm sido bastante explorados no subproblema linear do fluxo de carga em função da importância destes para o êxito do processo iterativo (Galiana et al, 1993; Borges et al, 1996; Flueck \& Chiang, 1998; Chianotis \& Pai, 2000; Cheng \& Shen, 2006). Não faz parte deste trabalho o desenvolvimento de um pré-condicionador específico para o problema de interesse, e sim fazer uso eficiente de um pré-condicionador baseado em fatoração incompleta (ILU). Estes pré-condicionadores diferem com base na regra de eliminação de elementos não-nulos que surgem em posições antes ocupadas por elementos nulos durante o processo de fatoração. Cada elemento não-nulo pode ser eliminado em função da sua posição, do seu valor absoluto, ou de uma combinação destes. Aqui é usada a re- 
gra proposta por (Saad, 2003) que fixa uma tolerância $\tau$ e um número $\rho$ de elementos não-nulos permitidos nas linhas dos fatores incompletos; a cada etapa do processo de eliminação todos os elementos não-nulos inferiores a uma tolerância relativa obtida pelo produto da tolerância absoluta $\tau$ pela norma-2 da linha correspondente são eliminados; e os restantes pelo menos os $\rho$ maiores em magnitude são mantidos. Este pré-condicionador é conhecido como $\operatorname{ILUT}(\tau, \rho)$.

Um pré-condicionador ILU pode apresentar vantagens e desvantagens desde a sua construção até a sua aplicação. As principais vantagens estão associadas à simplicidade e versatilidade para diferentes tipos de problema, menor custo computacional para construção, armazenamento e aplicação. Por outro lado, está sujeito a problemas de estabilidade numérica e de imprecisão dos seus fatores incompletos, geralmente associados à fatoração envolvendo elementos pivôs muito pequenos (Chow \& Saad, 1997), resultando em um pré-condicionador de baixa qualidade reduzindo a taxa de convergência do método iterativo. Apesar destas desvantagens, as suas vantagens o tornam atrativo para o problema de interesse, destacando a sua capacidade de adaptação para diferentes níveis de carga. Para fazer uso eficiente do précondicionador ILU é necessário melhorar as propriedades da matriz de coeficientes usada para a sua construção, neste caso a matriz Jacobiana. Os procedimentos para atingir tal objetivo são apresentados na próxima subseção.

\subsection{Convergência do GMRES}

\section{- Influência do Reordenamento}

Pré-condicionadores ILU são sensíveis ao reordenamento da matriz de coeficientes, reduzindo o número de elementos não-nulos e aumentando a robustez (estabilidade numérica) do processo de cálculo dos fatores triangulares incompletos. Normalmente, o reordenamento aumenta a taxa de convergência de métodos no subespaço Krylov (Benzi \& Tuma, 2000) e já foi testado na solução do problema de interesse. Em (Alves et al, 1999) foi proposto um esquema de reordenamento envolvendo matrizes simétricas definidas positivas que, segundo os autores, é tão eficiente para os métodos iterativos quanto o Tinney-2 é para os diretos. No presente trabalho é usada a estratégia Cuthill-McKee reverso - RCM, (Cuthill \& McKee, 1972), já considerada em (Flueck \& Chiang, 1998) na construção de um pré-condicionador ILU também para solução do fluxo de carga. Os autores se limitaram apenas em aplicar esta estratégia, mas através de análises matemáticas e computacionais é possível investigar o efeito do reordenamento na taxa de convergência do GMRES. Para isso, considere o subproblema linear do fluxo de carga solucionado na k-ésima iteração Newton-Raphson representado por (2) (Monticelli, 1983) e o processo de reordenamento por (3), onde P é a matriz de permutação composta por 1's e 0's (Cuthill \& McKee, 1972). A solução $\Delta \theta \mathrm{V}_{k}$ passa a ser representada pelo vetor $\Delta \mathrm{Z}_{k}$ reordenado (4).

$$
\begin{gathered}
\underbrace{\left[\begin{array}{cc}
{\left[\frac{d \Delta P}{d \theta}\right]} & {\left[\frac{d \Delta P}{d V}\right]} \\
{\left[\frac{d \Delta Q}{d \theta}\right]} & {\left[\frac{d \Delta Q}{d V}\right]}
\end{array}\right]}_{J_{k}} \cdot \underbrace{\left[\begin{array}{c}
\Delta \theta \\
\Delta V
\end{array}\right]^{k}}_{\Delta \theta V_{k}}=\left[\begin{array}{c}
\Delta P \\
\Delta Q
\end{array}\right]^{k} \\
{\left[P^{T} . J_{k} \cdot P\right] \cdot\left[\Delta Z_{k}\right]=P^{T} \cdot\left[\begin{array}{c}
\Delta P \\
\Delta Q
\end{array}\right]^{k}} \\
\Delta \theta V_{k}=P \cdot\left[\Delta Z_{k}\right]
\end{gathered}
$$

Com o sistema linear (4) pré-condicionado pela esquerda por $M_{k}^{-1}$ a solução passa a ser calculada por (5). O précondicionador não é construído explicitamente, sendo mais eficiente calcular seus fatores triangulares incompletos $\left(\bar{L}_{k}\right.$ e $\bar{U}_{k}$ ) por (6) usando as regras do pré-condicionador ILUT. Quanto mais o pré-condicionador se aproximar da inversa da matriz Jacobiana reordenada, mais o produto entre estas matrizes se aproxima de uma matriz identidade. Conseqüentemente, o sistema linear (4) é transformado em outro mais fácil de ser solucionado.

$$
\begin{gathered}
\underbrace{M_{k}^{-1} \cdot\left[P^{T} \cdot J_{k} \cdot P\right]}_{A_{k}} \cdot\left[\Delta Z_{k}\right]=\underbrace{M_{k}^{-1} \cdot P^{T} \cdot\left[\begin{array}{c}
\Delta P \\
\Delta Q
\end{array}\right]}_{b_{k}} \\
M_{k}=\bar{L}_{k} \cdot \bar{U}_{k} \cong P^{T} \cdot J_{k} \cdot P
\end{gathered}
$$

A cada iteração $i$ do método GMRES, a matriz $A_{k} 3$ é multiplicada por um vetor $v_{i}$ do subespaço Krylov, e também pela solução calculada $x_{i}$. Neste último caso, o resultado é usado para calcular o resíduo $r_{i}$, como apresentado em (7). A importância de se calcular o resíduo com uma boa precisão numérica está no fato deste ser usado no teste de convergência do método GMRES. Tanto o reordenamento quanto o pré-condicionamento influenciam o cálculo do resíduo.

$$
r_{i}=\underbrace{M_{k}^{-1} \cdot P^{T} \cdot\left[\begin{array}{c}
\Delta P \\
\Delta Q
\end{array}\right]^{k}}_{b_{k}}-\underbrace{M_{k}^{-1} \cdot\left[P^{T} \cdot J_{k} \cdot P\right]}_{A_{k}} \cdot \underbrace{\left[\Delta Z_{i}\right]}_{x_{i}}
$$


Em (Olschowka \& Neumaier, 1996) foram apresentadas estratégias baseadas em escalonamento e permutações nãosimétricas e simétricas (reordenamento) para transformar o sistema linear original em outro equivalente, com a matriz passando a ser diagonal dominante, apresentando um melhor condicionamento e agrupamento dos autovalores. Alguns benefícios associados a este pré-processamento são: (a) elimina a possibilidade do aparecimento de elementos nulos na diagonal principal, evitando realizar pivotação durante a fatoração, otimizando o processo; (b) elimina a possibilidade do aparecimento de elementos muito pequenos na diagonal principal, melhorando a precisão do cálculo dos elementos dos fatores incompletos; (c) torna os vetores coluna da matriz de coeficientes mais linearmente independentes, eliminando qualquer tipo de singularidade; (d) aumenta a estabilidade numérica (diminuição do erro de arredondamento) durante a fatoração. Estas estratégias foram implementadas eficientemente por (Duff \& Koster, 1999; 2001) para sistemas esparsos, testadas em métodos diretos e iterativos précondicionados para diferentes problemas (fluxo de carga não incluído). Para os casos do subproblema linear do fluxo de carga abordados neste trabalho, observou-se que a aplicação deste conjunto de estratégias só foi necessária em um caso extremo no qual o reordenamento não foi suficiente para gerar um pré-condicionador de qualidade e o método GMRES falhou no início do processo de solução. Portanto, em caso de falha devido a um pré-condicionador de baixa qualidade resultante de uma matriz mal-condicionada, esta deve ser préprocessada em quatro etapas, sendo estas:

i Identificação da seqüência de permutações que deslocará os maiores elementos de cada coluna da matriz Jacobiana até a diagonal principal. O produto destes elementos é conhecido como Máximo Produto Transversal (MPT) (Duff \& Koster, 1999; 2001).

ii A matriz de Jacobiana é escalonada estabelecendo-se que, os valores absolutos dos maiores elementos de cada coluna, identificados no passo (i), sejam iguais a um, e os restantes menores que um.

iii São realizadas as permutações não simétricas estabelecidas em (i) pela seqüência de permutações.

iv São realizadas as permutações simétricas (reordenamento).

Adaptando as etapas (i)-(iv) ao subproblema linear do fluxo de carga, o cálculo do resíduo dado por (7) passa a ser efetuado por (8), onde $D_{1}^{k}$ e $D_{2}^{k}$ são respectivamente, as matrizes de escalonamento por fila e coluna, $\mathrm{Q}_{k}$ é a matriz que efetua o deslocamento dos maiores elementos de cada coluna para posições da diagonal principal (permutações não-simétricas), e P, já apresentada, é a matriz de reordenamento (permutações simétricas).

$$
\begin{aligned}
r_{i}=\underbrace{M_{k}^{-1} \cdot P^{T} \cdot D_{1}^{k} \cdot\left[\begin{array}{c}
\Delta P \\
\Delta Q
\end{array}\right]^{k}}_{b_{k}}- \\
\underbrace{M_{k}^{-1} \cdot\left[P^{T} \cdot D_{1}^{k} \cdot J_{k} \cdot D_{2}^{k} \cdot Q_{k} \cdot P\right]}_{A_{k}} \cdot \underbrace{\left[\Delta Z_{i}\right]}_{x_{i}}
\end{aligned}
$$

Um conjunto preliminar de experimentos numéricos ilustra a influência dessas estratégias na taxa de convergência do método GMRES. Para isso, fluxos de carga foram simulados usando o sistema-teste IEEE-118 para as seguintes condições: sem pré-condicionamento (GMRES), com pré-condicionamento e sem reordenamento (GMRES+ILUT), com pré-condicionamento e reordenamento (GMRES+ILUT+RCM), e com pré-condicionamento e préprocessamento (GMRES+ILUT+PP). Os gráficos da Figura 1 mostram para estas condições a norma do resíduo associada à última iteração linear convergida e a taxa de convergência do GMRES. A norma é calculada de acordo com (9) para os resíduos dados por (7) e (8) e a taxa de convergência com (10) (Saad, 2003), onde $\mathrm{r}_{1}$ e $\mathrm{r}_{N i}$ são os resíduos da primeira e da última iteração linear convergida e $\mathrm{N}_{i}$ é o número total de iterações lineares. A Tabela 1 informa o número em milhões de operações de ponto flutuante (MFLOPs) para cada um dos experimentos numéricos. Os resultados indicam que o reordenamento melhora a norma do resíduo, a taxa de convergência e reduz o número de operações efetuadas, melhorando a robustez e eficiência do método GMRES.

$$
\begin{gathered}
\left\|r_{i}\right\|_{2}=\left\|b_{k}-A_{k} \cdot x_{i}\right\| \\
T C=\frac{\log _{10}\left\|r_{1}\right\| /\left\|r_{N i}\right\|}{N_{i}-1}
\end{gathered}
$$

Tabela 1: Operações de ponto flutuante

\begin{tabular}{|c|c|}
\hline Condição & MFLOPs \\
\hline GMRES & 534.15 \\
\hline GMRES+ILUT & 1.19 \\
\hline GMRES+ILUT+RCM & 0.053 \\
\hline GMRES+ILUT+PP & 0.056 \\
\hline
\end{tabular}



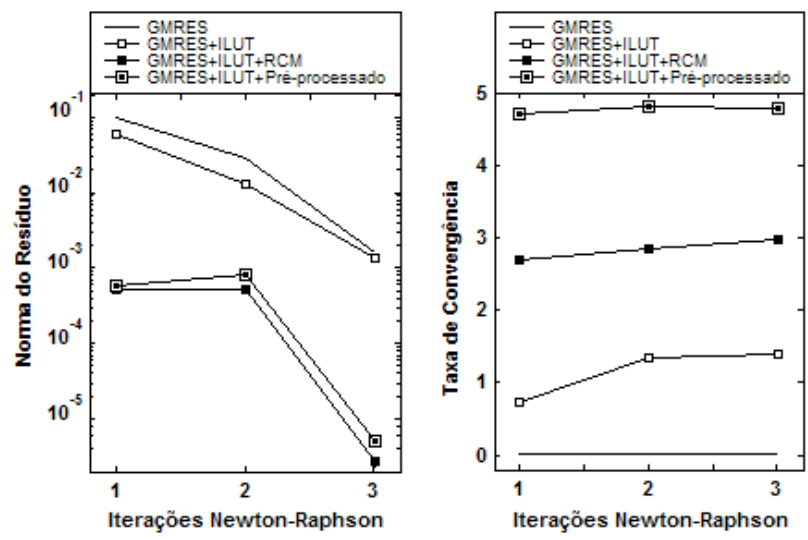

Figura 1: Norma do resíduo e taxa de convergência

\subsection{Indicadores de Qualidade}

É possível avaliar a precisão do pré-condicionador através da distância que o produto dos fatores incompletos está da matriz A, dada por (11). Já a estabilidade numérica pode ser medida pela distância que a matriz pré-condicionada está da matriz identidade, representada por (12) quando o pré-condicionamento é realizado pela esquerda, e por (13) quando realizado pela direita (Benzi, 2002). Essas análises feitas pelas normas de Frobenius são importantes para se trabalhar na qualidade do pré-condicionador e verificar se o reordenamento ou o pré-processamento da matriz Jacobiana surtiu o efeito esperado. Como neste trabalho o pré-condicionamento é realizado pela esquerda, as normas (11) e (12) são usadas na seção de experimentos numéricos apenas como "ferramentas" de análise da qualidade do précondicionador, não fazendo parte do processo de solução.

$$
\begin{gathered}
N_{1}=\|A-\bar{L} \cdot \bar{U}\|_{F} \\
N_{2}=\left\|I-(\bar{L} \cdot \bar{U})^{-1} \cdot A\right\|_{F} \\
N_{2}=\left\|I-A \cdot(\bar{L} \cdot \bar{U})^{-1}\right\|_{F}
\end{gathered}
$$

\section{EXPERIMENTOS NUMÉRICOS}

Investiga-se se a metodologia proposta afeta positivamente a qualidade dos pré-condicionadores, tornando o GMRES mais robusto. O solucionador direto MA28 (Duff, 1977; Gill et al, 2005; George \& Liu 1989; Oldenburg et al, 2000) que faz uso de fatoração LU e reordenamento minimum degree também participa dos experimentos. Foram usados os valores $10^{-3}$ e $10^{-5}$ como tolerâncias para os sistemas nãolineares e lineares, respectivamente. Como estimativa inicial do vetor de incógnitas necessário para a inicialização do GMRES, usou-se um vetor de elementos nulos. As simulações foram realizadas em um computador com processador Intel Centrino de $1.73 \mathrm{GHz}, 1 \mathrm{~GB}$ de RAM.

\subsection{Configuração I}

É considerada uma configuração do sistema interligado Brasileiro com 2256 barras, 3508 ramos e 200 geradores, três níveis de carga (Tabela 2) sem limitadores de potência reativa dos geradores e dos compensadores. Com relação aos parâmetros do ILUT, a escolha dos valores ótimos para $\rho$ e para a tolerância $\tau$ foi feita por tentativa e erro (Benzi,2002). Aqui, os valores encontrados após várias simulações de fluxo de carga com base no Caso I foram $\left(35,10^{-4}\right)$ usando o tempo de CPU como critério de decisão (quanto menor, melhor). Um simples laço de programação facilitou esta procura.

Tabela 2: Níveis de Carga

\begin{tabular}{|c|c|}
\hline Caso I & $49513 \mathrm{MW}$ \\
\hline Caso II & $50963 \mathrm{MW}$ \\
\hline Caso III & $50982 \mathrm{MW}$ \\
\hline
\end{tabular}

\subsubsection{Qualidade dos Pré-condicionadores}

Os Casos I e II só foram solucionados com reordenamento e o Caso III com a matriz Jacobiana pré-processada. A Figura 2 mostra a sua estrutura antes (a), e depois do reordenamento (b), onde se pode notar que os elementos nãonulos estão confinados numa banda estreita. Já a Figura 3(a) mostra a distribuição dos autovalores da matriz Jacobiana do Caso III sem pré-processamento onde se nota o seu maucondicionamento, justificando parcialmente a falha do método iterativo já que a matriz pode apresentar outras características que afetam negativamente a solução do problema (seção 2). Uma vez pré-processada, os autovalores da matriz Jacobiana se agrupam próximos da origem como mostra a Figura 3(b), melhorando as suas propriedades espectrais e a qualidade do pré-condicionador. Não foi possível considerar o pré-processamento para o solucionador direto MA28, pois o seu código fonte não está disponível.

As normas de Frobenius dadas por (11) e (12) oferecem uma melhor avaliação do efeito das estratégias na precisão e na estabilidade numérica dos fatores incompletos e completos dos pré-condicionadores. Estas normas não são necessárias no processo de solução do subproblema linear, sendo calculadas apenas para indicar a qualidade do pré-condicionador. Os valores médios dessas normas estão informados nas Tabelas 3 e 4 e quanto mais próximos de zero, melhor a qualidade do pré-condicionador. A coluna rotulada como "NV" corres- 


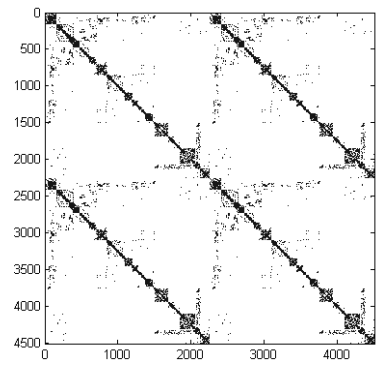

(a) Ordenamento Natural

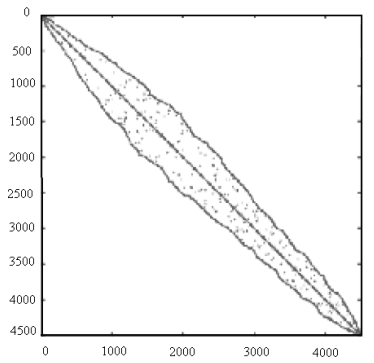

(b) Reordenada - RCM
Figura 2: Padrão de esparsidade da matriz Jacobiana

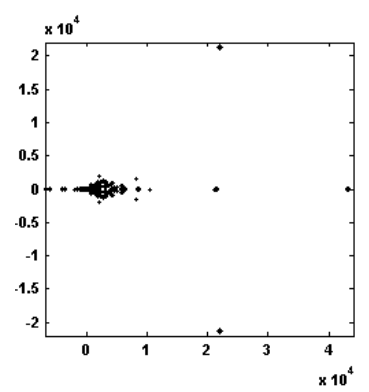

(a) Ordenamento Natural

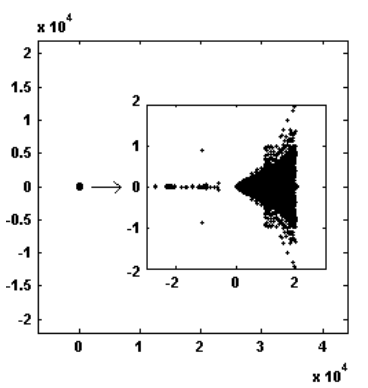

(b) Pré-processada
Figura 3: Distribuição Espectral dos Autovalores da Matriz Jacobiana - Caso III

ponde a construção de um novo pré-condicionador a cada iteração Newton-Raphson e a como "QF" apenas se necessário (Quase-Fixo). A norma $\mathrm{N}_{1}$ com ordenamento natural (sem reordenamento) é muito grande $\left(10^{17}\right) \mathrm{e}$, portanto, a degradação da qualidade do pré-condicionador e conseqüente falha do GMRES não são causadas pela instabilidade numérica dos fatores incompletos $\left(\mathrm{N}_{2}\right)$, e sim pela baixa precisão destes. O reordenamento e o pré-processamento reduzem substancialmente ambas as normas, sendo mais significativo para $\mathrm{N}_{1}$, melhorando a qualidade dos pré-condicionadores. Para LU com ordenamento natural o pré-condicionador não foi construído devido a problemas de espaço de memória.

A Tabela 5 informa o número de MFLOPs, de iterações lineares e tempo de CPU (em segundos) para cada caso simulado. Estas grandezas estão associadas apenas a solução do subproblema linear do fluxo de carga. Já os gráficos da Figura 4 mostram os ganhos e as perdas em porcentagem dos solucionadores iterativos em relação ao direto para os Casos I e II. A eficiência computacional do ILUT-GMRES fazendo uso da estratégia QF foi superior a apresentada pelos demais solucionadores. Esse aspecto é interessante uma vez que, a priori, a matriz Jacobiana não é grande o bastante para que o método iterativo superasse o direto em termos de eficiência computacional. Ainda, fazendo-se uma comparação entre as
Tabela 3: Pré-condicionador ILUT

\begin{tabular}{|c|c|c|c|c|c|}
\hline \multirow{2}{*}{ Condição de } & \multirow{2}{*}{$\mathrm{ON}^{1}$} & \multicolumn{2}{|c|}{ RCM $^{2}$} & \multicolumn{2}{|c|}{ P-P } \\
\cline { 2 - 6 } Operação & $N_{1}$ & $N_{1}$ & $N_{1}$ & $N_{1}$ & $N_{1}$ \\
& $N_{2}$ & $N_{2}$ & $N_{2}$ & $N_{2}$ & $N_{2}$ \\
\hline Caso I & $10^{17}$ & $2 \times 10^{4}$ & $2 \times 10^{4}$ & - & - \\
$49513 \mathrm{MW}$ & 156.4 & 74.5 & 90.1 & & \\
\hline Caso II & $10^{17}$ & $2 \times 10^{4}$ & $2 \times 10^{4}$ & - & - \\
$50963 \mathrm{MW}$ & 156.8 & 112.8 & 78.04 & & \\
\hline \multirow{2}{*}{ Caso III } & $10^{17}$ & $\begin{array}{c}2.5 \times \\
10^{9}\end{array}$ & $\begin{array}{c}3.1 \times \\
10^{9}\end{array}$ & 0.02 & 0.42 \\
& & $1.6 \times$ & $2.6 \times$ & 6.5 & 92.4 \\
\hline
\end{tabular}

${ }^{1}$ Ordenamento Natural, ${ }^{2}$ Reordenamento Cuthill-McKee reverso, ${ }^{3}$ Pré-Processamento.

Tabela 4: Pré-condicionador LU

\begin{tabular}{|c|c|c|c|c|}
\hline \multirow{2}{*}{ Condição de } & \multicolumn{2}{|c|}{ RCM } & \multicolumn{2}{c|}{ P-P } \\
\cline { 2 - 5 } & NV & QF & NV & QF \\
\cline { 2 - 5 } Operação & $N_{1}$ & $N_{1}$ & $N_{1}$ & $N_{1}$ \\
& $N_{2}$ & $N_{2}$ & $N_{2}$ & $N_{2}$ \\
\hline Caso I & 30.0 & 210 & & - \\
49513 MW & 0.0 & 61.3 & - & - \\
\hline Caso II & 30.0 & $3.2 \times 10^{3}$ & - & - \\
50963 MW & 0.0 & 74.3 & & \\
\hline \multirow{2}{*}{ Caso III } & $3.6^{17}$ & $2.5 \times 10^{9}$ & $8 \times$ & $10^{-3}$ \\
50982 MW & $1.5 \times 10^{2}$ & $1.6 \times 10^{2}$ & 0.0 & 37 \\
\hline
\end{tabular}

normas apresentadas nas Tabelas 3 e 4 nota-se que a qualidade do pré-condicionador LU é superior a do ILUT, resultando em menos iterações lineares efetuadas pelo GMRES. Entretanto, de acordo com os resultados da Tabela 5, os custos computacionais associados a sua construção e aplicação superam aos do ILUT, como se nota pelos maiores números de MFLOPs e maiores tempos de CPU.

\subsection{Configuração II}

O sistema-teste usado é outra configuração do sistema elétrico Brasileiro com 3515 barras, 5024 ramos, 301 geradores, apenas um nível de carga de 63857 MW (Caso IV) e os limites de potência reativa dos geradores e compensadores são considerados. Um novo valor ótimo para a tolerância $\tau$ foi encontrado de acordo com o procedimento ante- 

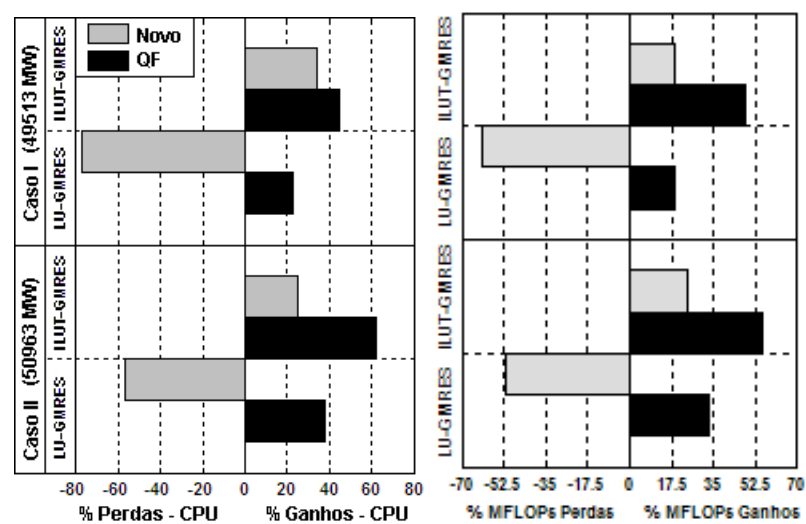

Figura 4: Ganhos e perdas -tempo de CPU e MFLOPs.

rior, resultando em $10^{-5}$. Mantendo o pré-condicionador fixo os solucionadores iterativos falharam em resolver o subproblema linear. Entretanto, construindo um novo a cada iteração Newton-Raphson, ou sempre que necessário, o caso foi solucionado pelos iterativos apenas com reordenamento. A estratégia de se construir um pré-condicionador quando necessário tira vantagem de um mecanismo inerente ao modelo de fluxo de carga considerado aqui (Monticelli, 1983). Quando os limites de geração de potência reativa dos geradores e compensadores são atingidos (ou deixam esta região), as correspondentes barras do tipo PV passam a ser do tipo PQ (ou vice-versa), grandes elementos são introduzidos (ou retirados) da diagonal principal da matriz Jacobiana atual e uma vez que o pré-condicionador disponível foi construído a partir de uma matriz diferente, sua qualidade é baixa para solucionar de forma eficiente o atual sistema linear, demandando por um pré-condicionador de melhor qualidade. A Figura 5 mostra a taxa de convergência do ILUT-GMRES. Após a $4^{a}$ iteração Newton-Raphson 96 geradores atingiram seus respectivos limites de potência reativa resultando num pré-condicionador de baixa qualidade na $5^{a}$ iteração e se for mantido fixo (FX) a taxa de convergência do GMRES vai a zero e a simulação é interrompida (número de iterações lineares excedido). Construindo um novo pré-condicionador a cada iteração Newton-Raphson (NV), ou de acordo com o mecanismo descrito (QF), o GMRES soluciona o problema sem apresentar o fenômeno da estagnação, caracterizando uma forte robustez numérica.

\section{CONCLUSÕES}

Este artigo mostrou que um pré-condicionador ILU pode ser muito eficiente e melhorar a taxa de convergência do método GMRES se problemas de precisão e de estabilidade numérica dos seus fatores incompletos provenientes do mau-condicionamento da matriz Jacobiana usada para a sua construção forem amenizados. Para se conseguir um pré-

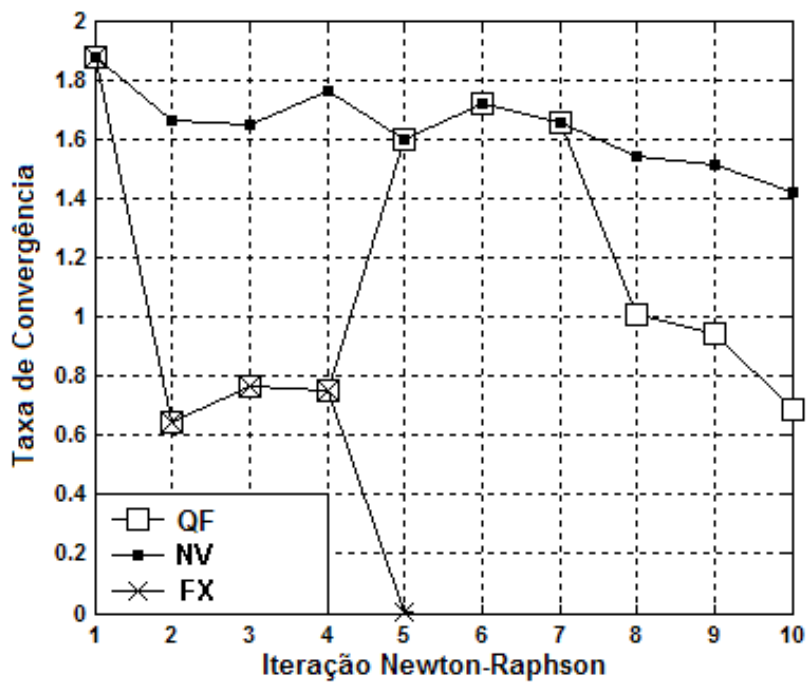

Figura 5: Taxa de convergência do ILUT-GMRES

condicionador ILU com tais qualidades, foi proposta uma metodologia que consiste em reordenar a matriz Jacobiana e tentar solucionar iterativamente o subproblema linear do fluxo de carga através do GMRES. Se este falhar devido à baixa qualidade do pré-condicionador, o processo é reiniciado e a matriz Jacobiana usada para a sua construção é pré-processada através de escalonamento, permutações nãosimétricas e simétricas (reordenamento) a fim de melhorar a qualidade do pré-condicionamento. Esta metodologia quando aplicada com o pré-condicionamento ILU QuaseFixo torna o método GMRES competitivo no aspecto eficiência computacional para solucionar problemas de difícil convergência, principalmente os cenários de carga pesada, como corroborado pelos resultados dos experimentos numéricos.

\section{REFERÊNCIAS}

Alvarado, F.L. (1979). Parallel Solution of Transient Problems by Trapezoidal Integration. IEEE Transactions on Power Apparatus and Systems, Volume PAS-98, Issue 3, May, Page(s): $1080-1090$.

Alves, A.B., Asada, E.N. \& Monticelli, A. (1999). Critical Evaluation of Direct and Iterative Methods for solving $\mathrm{Ax}=\mathrm{b}$ systems in Power Flow Calculations and Contingency Analysis. IEEE Transactions on Power Systems, Vol.14, $\mathrm{N}^{\circ} 2$.

Benzi, M. (2002), Preconditioning Techniques for Large Linear Systems: A Survey, Journal of Computation Physics, 182, pp. 418-477.

Borges, C.T., Coutinho, A.L.G.A. \& Falcão, D.M. (1996). Solução de Fluxo de Potência em Ambiente Vetorial 
Tabela 5: Resultados dos Experimentos Numéricos - Configuração I

\begin{tabular}{|c|c|c|c|c|c|}
\hline CASO I & \multicolumn{5}{|c|}{$49513 \mathrm{MW}$} \\
\hline Método & MA28 & \multicolumn{2}{|c|}{ ILUT-GMRES } & \multicolumn{2}{|c|}{ LU-GMRES } \\
\hline Estratégia & - & $\mathrm{NV}$ & $\mathrm{QF}$ & $\mathrm{NV}$ & $\mathrm{QF}$ \\
\hline MFLOPs & 3.0435 & 2.4602 & 1.5591 & 4.9205 & 2.4602 \\
\hline $\begin{array}{l}\text { Iterações } \\
\text { GMRES }\end{array}$ & - & 6 & 7 & 3 & 5 \\
\hline $\mathrm{CPU}$ & $0.141(\mathrm{~s})$ & $0.093(\mathrm{~s})$ & $0.078(\mathrm{~s})$ & $0.250(\mathrm{~s})$ & $0.109(\mathrm{~s})$ \\
\hline CASO II & \multicolumn{5}{|c|}{$50963 \mathrm{MW}$} \\
\hline Método & MA28 & \multicolumn{2}{|c|}{ ILUT-GMRES } & \multicolumn{2}{|c|}{ LU-GMRES } \\
\hline Estratégia & - & NV & $\mathrm{QF}$ & NV & $\mathrm{QF}$ \\
\hline MFLOPs & 5.0059 & 3.7906 & 2.2311 & 7.5953 & 3.3472 \\
\hline $\begin{array}{l}\text { Iterações } \\
\text { GMRES }\end{array}$ & - & 10 & 14 & 5 & 13 \\
\hline $\mathrm{CPU}$ & $0.249(\mathrm{~s})$ & $0.187(\mathrm{~s})$ & $0.094(\mathrm{~s})$ & $0.390(\mathrm{~s})$ & $0.155(\mathrm{~s})$ \\
\hline CASO III & \multicolumn{5}{|c|}{$50982 \mathrm{MW}$} \\
\hline Método & MA28 & \multicolumn{2}{|c|}{ ILUT-GMRES } & \multicolumn{2}{|c|}{ LU-GMRES } \\
\hline Estratégia & \multirow{4}{*}{ - } & NV & $\mathrm{QF}$ & NV & $\mathrm{QF}$ \\
\hline MFLOPs & & 9.6269 & 8.6109 & 18.6420 & 13.6719 \\
\hline $\begin{array}{l}\text { Iterações } \\
\text { GMRES }\end{array}$ & & 25 & 54 & 9 & 54 \\
\hline $\mathrm{CPU}$ & & $0.642(\mathrm{~s})$ & $0.594(\mathrm{~s})$ & $0.984(\mathrm{~s})$ & $0.735(\mathrm{~s})$ \\
\hline
\end{tabular}

usando o Método do Gradiente Bi-Conjugado Estabilizado. In: 11o. Congresso Brasileiro de Automática, São Paulo, Anais do 110. Congresso Brasileiro de Automática, v. 1. p. 160-166.

Borges, C.L., Falcão, D.M. \& Coutinho, A.L.G.A (1997). Utilização de Método Tipo Gradiente Conjugado na Aceleração do Fluxo de Potência em Computação Vetorial. In: XIV Seminário Nacional de Produção e Transmissão de Energia Elétrica, Belém.

Chai, J.S. \& Bose, A. (1993). Bottlenecks in parallel algorithms for power system stability analysis. Power Systems, IEEE Transactions on, Volume 8, Issue 1, Feb., Page(s): 9 - 15 .

Cheng, Y. \& Shen, C. (2006). A Jacobian-Free NewtonGMRES(m) Method with Adaptive Preconditioner and Its application for Power Flow Calculation. IEEE Transactions on Power Systems, Vol. 21, No. 3, pp. 10961103.
Chaniotis, D. \& Pai, M.A. (2000). Iterative Solver Techniques in the Dynamic Simulation of Power Systems. Power Engineering Society Summer Meeting, 2000. IEEE, Volume: 1, 609-613 vol. 1, Seattle, WA, USA.

Chow, E., \& Saad, Y. (1997). Experimental study of ILU preconditioners for indefinite matrices. J. Comput. Appl. Math. 86, vol. 2, pp. 387-414.

Cuthill, E. (1972). Several strategies for reducing the bandwidth of matrices. In: D.J. Rose and R.A. Willoughby, Editors, Sparse Matrices and their Applications, Plenum Press, New York, pp. 157-166.

Decker, I.C., Falcão, D.M. \& Kaszhurewicz, E. (1996). Conjugate Gradient Methods for Power System Dynamics Simulation on Parallel Computers. IEEE Transactions on Power Systems, vol.11, n³, pp 1218-1227.

Dong, W. \& Li, P. (2007). Hierarchical Harmonic-Balance Methods for Frequency-Domain Analog-Circuit Analysis. IEEE Transactions on Computer-Aided Design of Integrated Circuits and Systems, Vol. 26, No. 12, Dec. 
Duff, I.S. (1977). MA28. A set of Fortran subroutines for sparse unsymmetric linear equations. AERE Rep. R.8730, HMSO, London.

Duff, I.S. \& Koster, J. (1999). The design and use of algorithms for permuting large entries to the diagonal of sparse matrices. SIAM J. Matrix Anal. Appl. 20, 889.

Duff, I.S. \& Koster, J.K. (2001). On algorithms for permuting large entries to the diagonal of a sparse matrix. SIAM, J. Matrix Anal. Appl. 22, 973.

Flueck, A.J. \& Chiang, H.D. (1998). Solving the nonlinear power flow equations with an inexact Newton method using GMRES. IEEE Transactions on Power Systems, vol. 13 , no. 2 , pp. 267-273.

Galiana, F.D., Javidi, H., \& McFee, S. (1993). On the application of a preconditioned conjugate gradient algorithm to power network analysis. Proc. PICA Conference, pp. 404-410.

George, A. \& Liu, J.W. (1989). The Evolution of the Minimum Degree Ordering Algorithm. SIAM Review, Society for Industrial and Applied Mathematics, Vol. 31, No. 1 (Mar., 1989), pp. 1-19.

Gill, P.E., Murray, W. \& Saunders, M.A. (2005). SNOPT: An SQP Algorithm for Large-Scale Constrained Optimization. SIAM REVIEW, Society for Industrial and Applied Mathematics, Vol. 47, No. 1, pp. 99-131.

Lee, J., Zhang, J., \& Lu, C. (2004). Sparse inverse preconditioning of multilevel fast multipole algorithm for hybrid Integral equations in electromagnetic. Antennas and Propagation, IEEE Transactions on, Volume 52, Issue 9, Sept., Page(s): 2277 - 2287.

La Scala, M., Bose, A., Tylavsky, D.J. \& Chai, J.S. (1989). A highly parallel method for transient stability analysis. Power Industry Computer Application Conference, PICA '89, Conference Papers, 1-5 May, Page(s): 380 386.

Monticelli, A. (1983). Fluxo de carga em redes de energia elétrica. Editora Edgar Blücher Ltda, São Paulo, 1983.

Oldenburg C.M., Borglin S.E. \& Moridis G.J. (2000). Numerical Simulation of Ferrofluid Flow for Subsurface Environmental Engineering Applications. Kluwer Academic Publishers, Printed in the Netherlands, Transport in Porous Media 38: 319-344.

Olschowka, M. \& Neumaier, A. (1996). A new pivoting strategy for Gaussian elimination. Linear Algebra Its and Applications: 240, 131-151. Elsevier Science.
Pai, M.A., Sauer, P. W. and Kulkarni, A. Y.(1995) A Preconditioned Iterative Solver for Dynamic Simulation of Power System. 1995 IEEE International Symposium on Circuits and Systems, Volume: 2,pp. 1279-1282, Seattle, WA, USA.

Pai, M.A. \& Dag, H. (1997). Iterative Solver Techniques in large Scale Power System Computation. Proceedings of the 36th IEEE Conference on Decision and Control, page(s): 3861-3866 vol.4, San Diego, CA, USA.

Polydorides, N., Lionheart, W.R.B. \& McCann, H. (2002). Krylov subspace iterative techniques: on the detection of brain activity with electrical impedance tomography. Medical Imaging, IEEE Transactions on, Volume 21, Issue 6, June, Page(s): 596 - 603.

Saitoh, A. \& Kamitani, A. (2004). GMRES with new preconditioning for solving BEM-type linear system. Magnetics, IEEE Transactions on, Volume 40, Issue 2, Part 2, March, Page(s): 1084 - 1087.

Semlyen, A. (1996). Fundamental concepts of a Krylov subspace power flow methodology. IEEE Transactions on Power Systems, vol. 11, no. 3, pp. 1528-1537.

Saad, Y. \& Schultz, M.H. (1986). 'GMRES: a generalized minimal residual algorithm for solving nonsymmetric linear systems'. SIAM Journal of Scientific and Statistical Computing, vol.7, pp. 856-869.

Saad, Y. (2003). Iterative Methods for Sparse Linear System. 2nd edition, SIAM, Society for industrial and applied Mathematics, Philadelphia, PA.

Tinney, W. (1967). Direct solutions of sparse network equations by optimally ordered triangular factorization. Proceedings of the IEEE, Volume: 55, Issue: 11 , pp. 18011809 . 